\title{
CENTRIFUGAL-FLOW LVAD INFLOW CANNULA POSITION: PREOPERATIVE INFLUENCES AND POSTOPERATIVE OUTCOMES
}

\author{
Erik Sorensen ${ }^{1}$, Bartley Griffith ${ }^{2}$, Erika Feller ${ }^{2}$, Lynn Dees $^{3}$, and David Kaczorowski ${ }^{4}$ \\ ${ }^{1}$ University of Maryland Medical System \\ ${ }^{2}$ University of Maryland School of Medicine \\ ${ }^{3}$ University of Maryland Medical Center \\ ${ }^{4}$ University of Pittsburgh Medical Center
}

August 2, 2021

\begin{abstract}
Background: We previously demonstrated better inflow cannula (IFC) position and reduced pump thrombosis with a centrifugalflow LVAD (CF-LVAD) compared to an axial-flow device. We hypothesized that implant technique and patient anatomy would affect CF-LVAD IFC positioning and that malposition would impact LV unloading and outcomes. Methods: Preand postoperative computed tomography $(\mathrm{CT})$ scans were reviewed for patients with six-month follow-up. Malposition was quantified using angular deviation from an ideal line in two planes. IFC position was compared between conventional sternotomy (CS) and lateral thoracotomy-hemisternotomy (LTHS). The influence of LV end-diastolic dimension (LVEDD), body mass index (BMI), and CT-derived anatomy was determined. LV unloading was assessed by LVAD flow index (FI) and pre- to post-LVAD decrement in mitral regurgitation (MR) and LVEDD. Outcome measures were pump thrombus or stroke (PT/eCVA); 30day and total heart failure-related readmissions (HFRAs); and survival free of surgery for LVAD dysfunction. Results: One hundred fourteen patients met criteria. Total malposition magnitude was higher for CS than LTHS ( $\mathrm{p}=0.04)$. Midline-LV apex distance predicted lateral-plane malposition $(\mathrm{p}=0.04)$, while apex-LVOT angle predicted both anterior- $(\mathrm{p}=0.01)$ and lateral-plane $(\mathrm{p}=0.04)$ malposition. Lateral-plane malposition predicted decreased LVAD FI at three $(\mathrm{p}=0.03)$ and six $(\mathrm{p}=0.01)$ months. Total malposition magnitude predicted increased 30-day HFRAs $(\mathrm{p}=0.04)$, while lateral-plane malposition predicted more overall HFRAs $(\mathrm{p}=0.01)$. Malposition was not associated with PT/eCVA, changes in MR or LVEDD, or survival free of surgical revision. Conclusions: Patient anatomy and surgical technique were associated with CF-LVAD IFC malposition. In turn, malposition was associated with increased readmissions and decreased LVAD FI.
\end{abstract}

\section{INTRODUCTION}

We previously demonstrated better inflow cannula (IFC) positioning and less pump thrombosis with an intrapericardial centrifugal-flow LVAD (CF-LVAD: Heartware HVAD; Medtronic, Minneapolis MN) compared to an axial-flow device (Heartmate II; Abbott, Abbott Park, IL) ${ }^{1}$. Since that study, our default surgical approach for CF-LVAD insertion has evolved from conventional full sternotomy (CS) to a less invasive lateral thoracotomy/hemi-sternotomy (LTHS) approach ${ }^{2}$.

An advantage of LTHS is that the apical coring site can be selected and the sewing ring placed with the heart in its natural, undeformed position. For this reason, we hypothesized that LTHS would yield better IFC positioning than CS. We also assessed the influence of preoperative anatomic variables ${ }^{3}$.

Finally, we examined the effect of IFC position on left ventricle (LV) unloading and postoperative outcomes. LV unloading was assessed by LVAD flow index (FI = estimated LVAD flow/patient body surface area) 
and pre- to post-LVAD decrement in mitral regurgitation (MR) and LV end-diastolic dimension (LVEDD). Outcomes assessed were: pump thrombosis or embolic stroke (PT/eCVA); freedom from 30-day and total heart failure- and LVAD-related readmissions (HFRAs); and survival free of surgical intervention for malposition-related LVAD dysfunction.

\section{MATERIALS AND METHODS}

Medical records and imaging for adult patients undergoing implantation of a primary CF-LVAD with sixmonth follow-up as of January 31, 2021 were reviewed. Patients with durable BiVADs, non-apical cannulation, or lack of CTs were excluded. Data were collected prospectively in an IRB-approved database (University of Maryland IRB Protocol \#HP-00058592).

Before April 2016, the default technique was CS. Subsequently, the LTHS technique as described by $\mathrm{Schmitto}^{2}$, became the preferred approach. Intraoperative IFC position was assessed with transesophageal echocardiography. Hemodynamic and echo-guided LVAD optimization studies were also performed in the OR.

Preoperative CT scans were obtained for surgical planning and to rule out thoracic pathology. Postoperative scans were obtained to assess LVAD position. Contrast was given if permitted by renal function. Serial CT scans were not performed in stable patients. In patients with multiple scans, the most recent contrastenhanced CT was utilized.

Preoperative anatomic variables included LV end-diastolic dimension (LVEDD) and body mass index (BMI). We hypothesized that a smaller LVEDD would make optimal IFC placement more difficult. We hypothesized that extremes of BMI would contribute to malposition either by offering less intrathoracic space for the pump (low BMI) or by abdominal mass effect on the diaphragm (high BMI).

Preoperative CT measurements were: midline- LV apex distance in the axial plane (Figure 1A); and inclination of the LV apex-LV outflow tract axis above the horizontal (LVOT angle) in the coronal plane (Figure 1B). These measurements were derived from our prior work ${ }^{3}$, but we hypothesized different effects on IFC position for the CF-LVAD than observed for the axial-flow device. Since IFC position in the CF-LVAD tends toward horizontal, we expected a higher, rather than lower, LVOT angle to be associated with IFC malposition. For midline-LV apex distance, we expected a larger value to be associated with less space between apex and ribs, forcing the IFC downward and/or lateral.

Quantitative IFC position was calculated by measuring angular deviation from an axis intersecting the LV apex and the center of the mitral valve (MV). Angles in two orthogonal planes, anterior and lateral, aligned with this axis were measured using Aquarius iNtuition 4.4 (TeraRecon; Foster City, CA) (Figure 2). In the anterior plane, positive displacement signifies angulation toward the superior wall, and negative toward the inferior wall. In the lateral plane, positive displacement is toward the lateral wall, and negative toward the septum. Total malposition magnitude represents the sum of the magnitudes of the two angles.

LV unloading was assessed using the change in LVEDD and MR compared to the last pre-LVAD echocardiogram. MR was graded as none (0), mild (1), moderate (2), or severe (3). Both early (1-3 months post-LVAD) and late (6-12 months post-LVAD) measurements were used. LVAD FI was obtained from estimated LVAD flow $(\mathrm{L} / \mathrm{min})$ divided by patient BSA $\left(\mathrm{m}^{2}\right)$ at one-, three-, and six-month timepoints. Our institution does not routinely obtain invasive hemodynamic measurements on stable LVAD patients.

Postoperative outcomes included PT/eCVA, HFRAs and survival free of urgent transplant or explant for LVAD dysfunction, pump replacement, or pump repositioning.

Pump thrombosis was either thrombus identified within the LVAD at explant, or [?]2 of: increased lactate dehydrogenase ([?]1.5 $\times$ stable baseline or [?]2.5x upper normal) or plasma hemoglobin ([?]20 mg/dL); sustained abnormal power consumption ([?]+-1.5 W from stable baseline, or above manufacturer's suggested upper normal ${ }^{4}$ ); and new-onset clinical or hemodynamic signs of congestive heart failure. Embolic CVA was a focal neurologic deficit persisting $>24$ hours, or a new ischemic lesion on CT imaging. 
HFRAs were any readmission for heart failure symptoms or LVAD/hemodynamic derangements. This excluded infection and bleeding events, INR derangements, and elective admissions for planned diagnostic or surgical procedures. Prevalence of 30-day and overall HFRAs was assessed.

Statistical analysis was performed with Statistica 13 (Dell, Inc; Tulsa, OK). Normality of continuous variables was assessed with the Shapiro-Wilk test. Normally distributed variables are reported as mean +- standard deviation; non-normal as median (interquartile range). Categorical variables were compared with Fisher's exact test for $2 \times 2$ tables and the Pearson chi-squared test for higher dimensional tables. Association between preoperative anatomy and IFC angulation was assessed with linear regression. Association between cannula position and continuous outcome variables was also assessed with linear regression, while its relationship to dichotomous outcome variables was assessed with logistic regression. Cox proportional hazards regression was used to assess the effect of IFC position on survival free of need for surgical intervention. For all tests, a two-sided p-value $<0.05$ was considered significant.

\section{RESULTS}

During the study period, 114 patients met inclusion criteria. Table 1 lists baseline characteristics. Patients were predominantly male $(80 \%)$ and non-white $(62 \%)$ with non-ischemic cardiomyopathy $(70 \%)$. Median BMI was $28.0 \mathrm{~kg} / \mathrm{m}^{2}$, and median LVEDD was $6.8 \mathrm{~cm}$. Thirty-seven patients $(33 \%)$ were implanted via the CS technique and $77(67 \%)$ via LTHS. CT imaging revealed a mean midline-LV apex distance of $116.8 \mathrm{~mm}$ and a mean LVOT angle of 32.2 degrees. Median follow-up was 410 (194-788) days.

\section{Relationship of IFC Position to Surgical Approach}

IFC position by technique is depicted graphically in Figure 3 and quantitatively in Table 2. Anterior plane deviation was inferior wall-directed, with median -19.2 degrees for CS and -16.3 degrees for LTHS ( $\mathrm{p}=0.33)$. Lateral plane deviations were similar, with median 0.0 degrees for CS and 0.9 degrees for LTHS ( $\mathrm{p}=0.08)$. Total malposition magnitude was higher for CS (median 34.8 degrees) than LTHS (28.2 degrees; $\mathrm{p}=0.04$ ). Figure 3 shows more CS recipients with larger deviations in the infero-septal direction.

\section{Relationship of IFC Position to Preoperative Anatomy}

Table S1 (see Supplemental Material) presents univariate linear regression results for the association between preoperative anatomy and IFC position, while Figure 4 depicts the significant relationships. There was a negative relationship between LVOT angle and anterior-plane IFC angle (beta $=-0.534 ; \mathrm{p}=0.01$ ), indicating a steeper LVOT angle was associated with an inferiorly-directed IFC. Conversely, there was a positive relationship between LVOT angle and lateral-plane IFC angle (beta $=0.517, \mathrm{p}=0.04$ ), indicating a propensity toward laterally-directed IFCs with a more steeply tilted LVOT-apex axis. There was also a significant negative relationship between apex-midline distance and lateral plane IFC angle (beta $=-0.204, \mathrm{p}=0.04$ ), indicating that a more medial apex predicted lateral-wall malposition, while a laterally displaced apex was associated with a septally-oriented IFC. No anatomic variable predicted total malposition magnitude, and neither LVEDD nor BMI predicted any IFC malposition measure.

Figure 5 depicts the influence of anatomy on IFC position. The pre-LVAD CT demonstrates a more steeply inclined apex-LVOT axis (A: 35.2 degrees; median 32 degrees), while the post-LVAD CTA demonstrates IFC deviation in the inferior (B: -30.6 degrees) and lateral (C: +16.9 degrees) directions as predicted by the regression model.

\section{Effect of IFC Position on LV Unloading}

Table S2 lists the univariate linear regression results for the influence of IFC position on VAD FI, MR, LVEDD, and. Median values for each LV unloading-related variable are given for reference.

VAD FI data were available for 112, 107, and 95 patients at one, three, and six months. Median VAD FI ranged from $2.19-2.48 \mathrm{~L} / \mathrm{min} / \mathrm{m}^{2}$, decreasing over time. There was a negative association between lateral plane angle and LVAD FI at three $(\mathrm{p}=0.03)$ and six $(\mathrm{p}=0.01)$ months, indicating that an increasing angle predicts a lower flow index. 
When lateral-plane angle is binarized to lateral wall-directed (angle $>0 \mathrm{deg}$ ) or not, there is a significantly lower VAD FI observed at both three and six months in patients with laterally-directed IFCs (Figure 6; p $=0.002$ for both timepoints).

MR data were available for 83 patients in the early (1-3 month) and 85 patients in the late (6-12 month) timeframe. No patient underwent concomitant mitral valve repair with LVAD. MR versus pre-LVAD decreased by a median of one grade on both assessments. There was no association between IFC position measurements and MR decrement.

Finally, LVEDD was available for 84 patients in the early and 86 patients in the late window. LVEDD decreased by a median $11.7 \%$ on early and $6.5 \%$ on late assessment. There was no significant association between LVEDD decrement and any IFC position measurement.

\section{Effect of IFC Position on Outcomes}

Table S3 gives univariate logistic regression results for the influence of IFC position on PT/eCVA and freedom from HFRAs, as well as the Cox proportional hazards regression for survival free from surgical intervention for LVAD dysfunction. Prevalence of each outcome is given for reference.

Readmission data were available for 102 patients. Twenty percent had 30-day HFRAs, and $70 \%$ experienced one or more HFRAs during the follow-up period. There were significant relationships between IFC position and readmissions (Figure 7). A negative anterior plane angle predicted increased overall HFRAs ( $\mathrm{p}=0.01$ ), with an odds ratio of 3.35 for an angle [?] $-20 \mathrm{deg}(\mathrm{p}=0.01)$. Increasing total malposition magnitude predicted increased 30-day HFRAs $(\mathrm{p}=0.04)$, with an odds ratio of 3.65 for a deviation [?] $40 \operatorname{deg}(\mathrm{p}=$ $0.02)$.

Twenty-two percent of patients experienced PT/eCVA. There was no relationship between IFC position and $\mathrm{PT} / \mathrm{eCVA}$. There was also no influence of IFC position on survival free of reoperation for LVAD dysfunction.

\section{DISCUSSION}

This study expands upon our previous work quantitatively assessing IFC malposition ${ }^{1}$ and its relationship to preoperative anatomy ${ }^{3}$ and outcomes ${ }^{1}$. We believe this is one of the largest series utilizing three-dimensional CT data to systematically investigate factors influencing IFC position and its relationship to outcomes.

\section{Relationship of IFC Position to Surgical Approach}

We first compared IFC position after CF-LVAD insertion via CS or LTHS. Our hypothesis was that IFC position would improve with LTHS because the insertion site is determined with the heart in its natural position. We found that LTHS yielded less total malposition magnitude. As shown in Figure 3, this is primarily because several CS patients have larger infero-septal IFC deviations. With both approaches, the most common orientation of the IFC was below the apex-MV axis.

The results are consistent with Stoeckl et al. ${ }^{5}$, who found that a bilateral thoracotomy approach for CF-LVAD placement resulted in a lower IFC angle on chest radiograph than for CS.

Relationship of IFC Position to Preoperative Anatomy

We prospectively assessed anatomic variables that previously predicted Heartmate II IFC malposition. These included LVEDD, BMI, LV apex-midline distance, and LV apex-LVOT angle.

We did not find a relationship between LVEDD and malposition. A smaller LVEDD was associated with mortality and stroke in CF-LVADs ${ }^{6}$. We also found no association between malposition and PT/eCVA or mortality, as discussed below. Additional contributing factors beyond pump position likely explain this relationship.

We also found no relationship between BMI and malposition. A large INTERMACS registry study linked obesity with device malfunction and thrombosis, as well as readmissions ${ }^{7}$. However, only $16.8 \%$ of patients 
in that study received a CF-LVAD. It is possible that the smaller profile CF-LVADs makes them less sensitive to the effects of abdominal obesity than Heartmate II, which requires a pre- or intra-peritoneal pocket.

Both CT imaging measurements predicted malposition. A larger midline-LV apex distance was associated with increasingly septal IFC deviation. Additionally, a steeper LVOT angle predicted IFC deviation toward both the inferior wall and lateral walls.

A larger midline-LV apex distance indicates a more laterally and posteriorly displaced apex. This may increase the risk of malposition by making it difficult to reach the "true" apex. This is exacerbated in LTHS patients if the thoracotomy is not sufficiently lateral. Additionally, in these patients, the heart often impinges on the ribcage, so the pump can be displaced by the ribs at closure. By contrast, a shorter midline-LV apex distance predicted increased lateral-wall malposition. This measurement suggests an apex closer to midline, where the anterior chest wall could push the pump medially at closure.

A steeper LVOT angle indicates a more vertically-oriented heart. Since the natural lie of the CF-LVAD is horizontal, inserting it an up-tilted ventricle will tend to point it inferior to the apex-MV axis. The apex in these heart also tend to be more medial, which could portend a laterally displaced IFC by the mechanism discussed above.

Having become more cognizant of malposition risk, we routinely use preoperative CTs to select the intercostal space and extent of incision for LTHS procedures. We also obtain biplanar transesophageal echo imaging both before and after the chest is closed, since a uniplanar four-chamber view will miss inferior-wall malposition. We also have found preliminary success in repositioning CF-LVADs via a thoracotomy by pulling them inferiorly and securing them to a rib.

\section{Effect of IFC Position on LV Unloading and Outcomes}

We found that a laterally-directed IFC predicted significantly reduced VAD FI at three and six months (Figure 6). Computational studies ${ }^{8,9}$ have shown that increasingly lateral IFC position causes poor LV washout and longer particle residence times, consistent with poor LV unloading and compromised flow. Imamura ${ }^{10}$ found that increasing HVAD pump body area in the coronal x-ray plane was associated with a reduced decrement in LVEDD and pulmonary capillary wedge pressure during ramp studies. Larger pump area would correspond to a more laterally-oriented IFC.

In contrast, we did not find an association between malposition and decrement in MR or LVEDD from pre- to post-LVAD. However, we had only fixed-RPM echo studies. This MR finding is contrast to our previous work, wherein greater total malposition magnitude predict a higher degree of post-LVAD MR ${ }^{11}$. However, the studies differ on a few key aspects: a) Pasrija included only patients with at least mild-moderate preoperative MR; b) they used a subset of the current population (only patients implanted 2013-2017; $\mathrm{N}=$ 41 vs 83 patients in the current study); and c) MR was graded on a more granular scale, which captured intermediate degrees such as mild-moderate. These differences likely explain the discrepancy.

Imamura $^{10}$ also found that a steeper coronal plane IFC angle ([?] $76 \mathrm{deg}$ ) was associated with increased LVEDD and wedge pressure. However, such an angle represents relatively extreme superior-wall directed malposition, which was rare in our study (Figure 3). Imamura's report has only nine patients in this group and 27 total patients, so their findings may not be translatable to a larger population.

This and other clinical studies of IFC malposition in CF-LVADs ${ }^{10,12,13}$ rely on two-dimensional measurements of IFC angle relative to the spine or horizontal in the coronal plane, and are unable to identify septal or lateral-wall malposition.

We did find that IFC malposition was associated with readmissions (HFRAs). Patients with total malposition magnitude $>40 \mathrm{deg}$ had an increased incidence of 30-day HFRAs, while patients with a lateral plane angle [?] -20 deg experienced an increased incidence of overall HFRAs. Imamura associated a higher IFC coronal angle $(>65 \mathrm{deg})$ with increased HFRAs in HVAD patients ${ }^{10}$, while an angle $>28 \mathrm{deg}$ was the cut-off in a larger population with the Heartmate $3^{12}$. It is unclear why the two devices have such different thresholds, 
since both are intrapericardial with integral IFCs of roughly the same diameter. In contrast, Shih et al. ${ }^{13}$ found increased "malposition-related" adverse event rates, including HFRAs, in patients with a Heartmate 3 whose median spine-IFC angle on chest x-ray was $66 \mathrm{deg}$ at one month, compared to 59 deg in patients without adverse events. In this study, a higher pump-spine angle would correspond to a shallower angle using the method of Imamura.

Finally, we did not find an association between malposition and either PT/eCVA or survival free of malposition-related surgery. PT and eCVA have multifactorial causes, including hypertension and inadequate antithrombotic therapy ${ }^{14,15}$. While we did observe an association between malposition and pump thrombosis in the Heartmate II, we observed deviations that were much more extreme than those observed for the HVAD, and which tended to be primarily superior-wall and septum-directed ${ }^{1}$. The degree of malposition observed here may not be sufficient to independently cause thrombosis. Mortality is similarly multifactorial.

\section{LIMITATIONS}

While we attempted to obtain imaging on all patients, $7 \%$ lacked CTs. Patients implanted urgently may not have preoperative imaging, while patients who expire or are transplanted during the index hospitalization may not have postoperative CTs. We also did not serially assess IFC position at pre-specified intervals. Follow-up scans were only obtained if patients had pump-related complications potentially related tode novo or worsening malposition. Shih ${ }^{13}$ found no major change in Heartmate 3 pump-spine angle on x-ray during 12-month follow-up.

We also did not assess LV unloading using invasive hemodynamics. Our center does not routinely obtain these studies outside of the initial hospitalization. We used LVAD FI as a surrogate for cardiac output, but did not have a similar measure for LV filling pressures. We also did not have serial echoes at pre-specified time points, and instead used two broad intervals: early (1-3 months post-LVAD) and late (6-12 months).

Finally, while the population studied $(\mathrm{N}=114)$ is one of the largest malposition series presented, these are single-center, single-device data covering a ten-year timespan. We chose to only include the HVAD in this series because we had extensive experience with and a well-established surgical protocol for that device. During the study period, only six Heartmate 3 patients met criteria, and we were still in the learning stages of using the LTHS technique for it. However, both pumps have similar profiles and similar-size integral IFCs so there is no reason to think our results would not apply to that device as well.

\section{CONCLUSIONS}

In this study, we extended our prior work on IFC malposition ${ }^{1,3}$ by assessing the effect of preoperative anatomic and surgical technique on CF-LVAD IFC malposition, and the effects of malposition on LV unloading and outcomes. We found that the LTHS technique was associated with a lower total malposition magnitude, and that a more laterally and posteriorly displaced apex and a more steeply inclined apex-LVOT axis portend malposition. Post-LVAD, we found that IFC malposition was associated with lower LVAD FI and with increased 30-day and overall HFRAs. Careful surgical planning and attention to intraoperative IFC positioning may mitigate these risks. Further study in a large, multicenter cohort is warranted.

\section{REFERENCES}

1. Sorensen EN, Kon ZN, Feller ED, Pham SM, Griffith BP: Quantitative Assessment of Inflow Malposition in Two Continuous-Flow Left Ventricular-Assist Devices Ann Thorac Surg 105: 1377-83, 2018

2. Schmitto JD, Molitoris U, Haverich A, Strueber M: Implantation of a centrifugal pump as a left ventricular assist device through a novel, minimized approach: upper hemisternotomy combined with anterolateral thoracotomy J Thorac Cardiovasc Surg 143: 511-513, 2012

3. Sorensen EN, Hiivala NJ, Jeudy J, Rajagopal K, Griffith BP: Computed tomography correlates of inflow cannula malposition in a continuous-flow ventricular-assist device $J$ Heart Lung Transpl 32: 654-7, 2013 
4. Chorpenning K, Brown MC, Voskoboynikov N, Reyes C, Dierlam AE, Tamez D: HeartWare controller logs a diagnostic tool and clinical management aid for the HVAD pump ASAIO J 60: 115-118, 2014

5. Stoeckl EM, Smith JW, Dhingra R, Fiedler AG: Left ventricular assist device implantation by bithoracotomy technique: A single-center perspective J Card Surg, 2021

6. Truong VT, Shreenivas S, Mazur W, et al.: Left Ventricular End-Diastolic Dimension and Clinical Outcomes After Centrifugal Flow Left Ventricular Assist Device Implantation ASAIO J Am Soc Artif Intern Organs 1992, 2021

7. Jaiswal A, Truby LK, Chichra A, et al.: Impact of Obesity on Ventricular Assist Device Outcomes J Card Fail , 2019

8. Chivukula VK, Beckman JA, Prisco AR, et al.: Left Ventricular Assist Device Inflow Cannula Angle and Thrombosis Risk Circ Heart Fail11: e004325, 2018

9. Neidlin M, Liao S, Li Z, et al.: Understanding the influence of left ventricular assist device inflow cannula alignment and the risk of intraventricular thrombosis Biomed Eng Online 20: 47, 2021

10. Imamura T, Nguyen A, Chung B, et al.: Association of Inflow Cannula Position with Left Ventricular Unloading and Clinical Outcomes in Patients with HeartMate II Left Ventricular Assist Device ASAIO J Am Soc Artif Intern Organs 1992 , 2018

11. Pasrija C, Sawan MA, Sorensen E, et al.: Inflow Cannula Position Influences Improvement in Mitral Regurgitation After Ventricular Assist Device Implantation ASAIO J Am Soc Artif Intern Organs 1992 67: 423-429, 2021

12. Imamura $\mathrm{T}$, Narang $\mathrm{N}$, Nitta $\mathrm{D}$, et al.: Optimal cannula positioning of HeartMate 3 left ventricular assist device Artif Organs 44: e509-e519, 2020

13. Shih H, Butler C, Melehy A, et al.: Serial assessment of HeartMate 3 pump position and inflow angle and effects on adverse events Eur J Cardiothorac Surg, 2021

14. Najjar SS, Slaughter MS, Pagani FD, et al.: An analysis of pump thrombus events in patients in the HeartWare ADVANCE bridge to transplant and continued access protocol trial $J$ Heart Lung Transpl 33: 23-34, 2014

15. Teuteberg JJ, Slaughter MS, Rogers JG, et al.: The HVAD Left Ventricular Assist Device: Risk Factors for Neurological Events and Risk Mitigation Strategies JACC Heart Fail 3: 818-828, 2015

\section{FIGURE LEGENDS}

Figure 1: Example of preoperative CT-derived anatomic measurements. A. Axial plane midline-to-LV apex distance. B. Coronal plane angulation of LV apex-LV outflow tract axis.

Figure 2: Example of inflow cannula (IFC) angular deviation measurements. Ideal line in each plane is from LV apex through approximate center of mitral valve. Actual position is line through IFC center. A. Anterior plane. Deviation is toward superior $(+)$ or inferior $(-)$ free wall. Illustrated cannula deviates very slightly inferiorly. B. Lateral plane. Deviation is toward septum (-) or lateral wall $(+)$. Illustrated cannula deviates very slightly septally.

Figure 3: CF-LVAD inflow cannula position by surgical technique. Open circles: conventional sternotomy. Black stars: lateral thoracotomy/hemisternotomy

Figure 4: Significant linear relationships between anatomic variables and IFC angles. Best-fit linear regression lines (solid) and 95\% confidence intervals (dashed) are shown. A. Anterior plane angle vs LVOT angle $(\mathrm{p}=0.01)$; B. Lateral plane angle as function of LVOT angle $(\mathrm{p}=0.04)$; C. Lateral plane angle as function of apex-midline distance $(\mathrm{p}=0.04)$. 
Figure 5: Example patient with higher LVOT angle (35.2 deg) on preoperative CT (A). Postoperative CT demonstrates (B) inferior-wall (-30.6 deg) and (C) lateral-wall directed (+16.9 deg) inflow malposition consistent with regression models.

Figure 6. Box and whisker plots of three-month (A) and six-month (B) VAD flow index (FI) stratified by whether inflow cannula is angulated toward lateral wall. $\mathrm{p}=0.002$ for both timepoints.

Figure 7. Box and whisker plots of relationship between readmission prevalence and inflow malposition. A. Anterior plane angle and overall heart failure/VAD-related readmissions (HFRAs; $p=0.01$ ). B. Total angular deviation and 30-day HFRAs $(\mathrm{p}=0.04)$.

Table 1

Variable

$(\mathrm{N}=114)$

Age (yr)

$56.5(44.9,63.0)$

Male, $\mathbf{n}(\%)$

91 (80)

White, n (\%)

43 (38)

Ischemic etiology, n (\%)

34 (30)

INTERMACS class, $\mathrm{n}(\%)$ :

1

$23(20)$

2

$27(24)$

3

$51(45)$

$[?] 4$

$13(11)$

BMI $\left(\mathrm{kg} / \mathrm{m}^{2}\right)$

28.0 (24.1, 33.8)

LV end-diastolic dimension (cm)

$6.8(6.1,7.8)$

LV ejection fraction (\%)

$15.0(12.5,17.5)$

Surgical approach (\% LTHS)

77 (67) 
Midline-to-LV apex distance $(\mathrm{mm})$

$116.8+-16.4$

Apex-to-LVOT midpoint angle (degrees)

$32.2+-6.5$

\section{Follow-up duration (days)}

410 (194-788)

Legend: Preoperative characteristics and surgical approach. Abbreviations: BMI: Body mass index; LTHS: left thoracotomy-hemisternotomy; LV: left ventricle; LVOT: LV outflow tract.

\section{Table 2}

\begin{tabular}{llll}
\hline Variable & $C S(N=37)$ & LTHS $(N=77)$ & p-value \\
\hline Anterior plane angle (degrees) & $-19.2(-28.3,-9.2)$ & $-16.3(-24.5,-9.2)$ & 0.33 \\
Lateral plane angle (degrees) & $0.0(-17.1,15.9)$ & $0.9(-3.2,11.9)$ & 0.08 \\
Total malposition magnitude (degrees) & $34.8(24.5,46.1)$ & $28.2(18.6,36.8)$ & $\mathbf{0 . 0 4}$ \\
\hline
\end{tabular}

Legend: Comparison of measures of quantitative malposition between surgical approaches.

Variables are displayed as median (interquartile range)

Abbreviations: CS: conventional sternotomy; LTHS: lateral thoracotomy/hemisternotomy
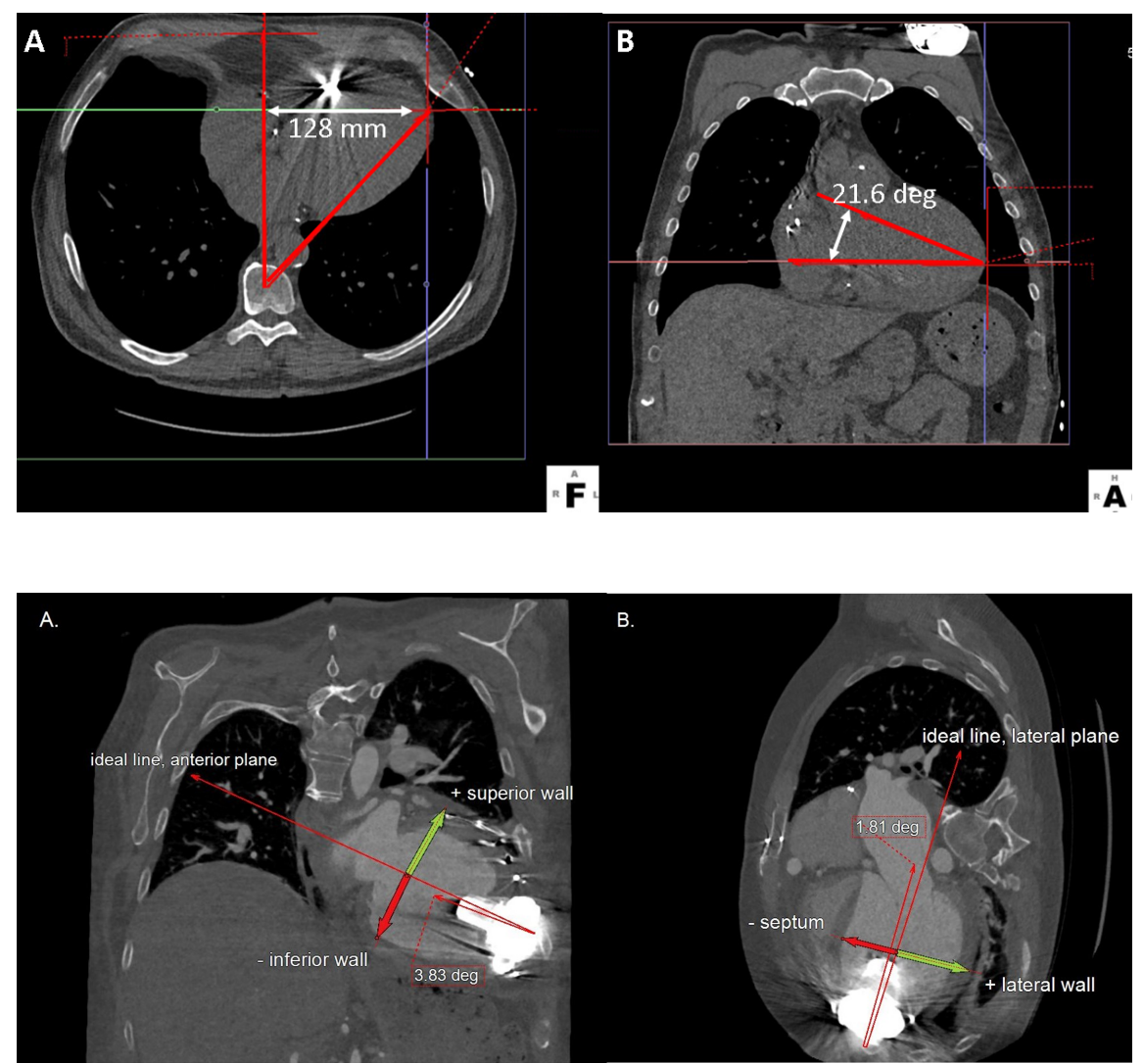

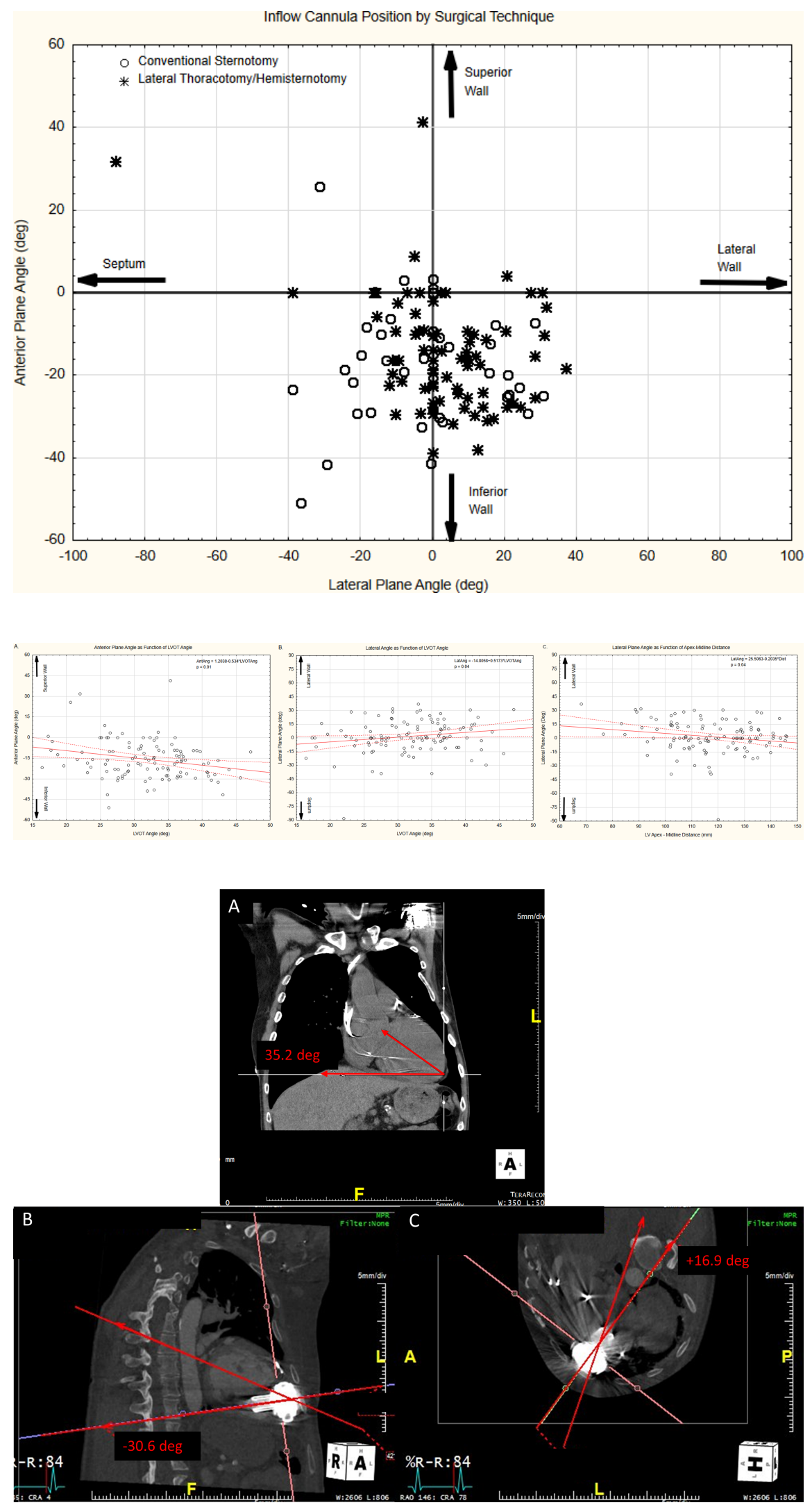

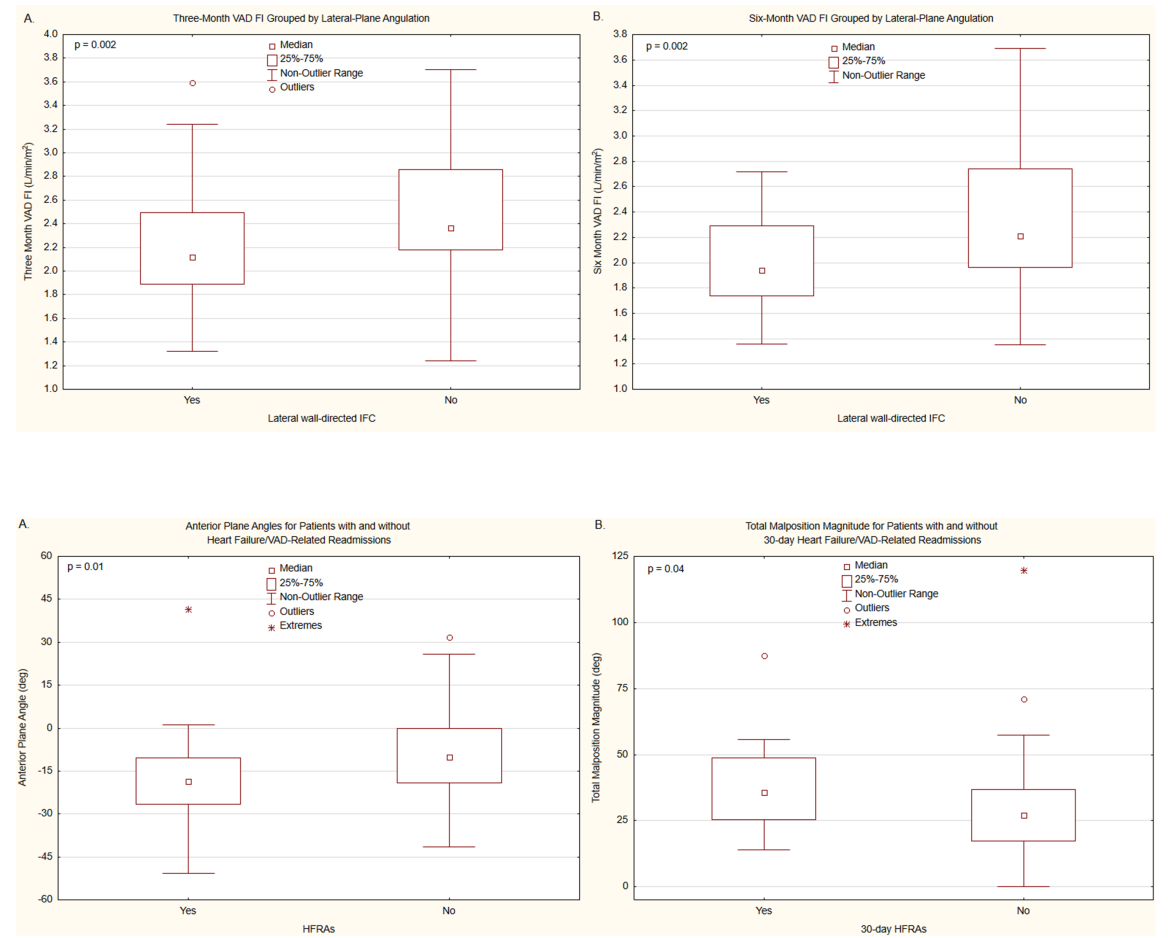